\title{
Do brain tissue transplants alter personal identity? Inadequacies of some "standard" arguments
}

\author{
Georg Northoff University of Frankfurt, Germany
}

\begin{abstract}
Currently, brain tissue transplantations are being developed as a clinical-therapeutic tool in neurodegenerative diseases such as Parkinson's or Alzheimer's disease. From an ethical point of view, distinguishing between the preservation and an alteration of personal identity seems to be central to determining the scope for further application of brain tissue transplantation therapy.

The purpose of this article is to review "standard" arguments which are used on the one hand by proponents to prove preservation of personal identity and by opponents on the other hand to prove that brain tissue transplantation results in an altered personal identity. Proponents and opponents are shown to use the same arguments, albeit with different presuppositions. These presuppositions concern the meaning of the term "identity", either numerical or qualitative, the definition of brain identity, either structurally or functionally, and the relationship between mental states, psychological functions and neurophysiological properties as criteria for personal identity. Furthermore the respective neurophysiological, clinical and philosophical evidence for the different presuppositions are discussed.

It is concluded that evaluation of personal identity in brain tissue transplantation should not only rely on the "standard" arguments but, additionally, neurophysiological, clinical and philosophical implications should be discussed.
\end{abstract}

\section{Introduction}

Currently only patients with impaired motor function as a result of Parkinson's disease are treated with brain tissue transplantation. Other diseases with rather psychological disturbances like Huntington's and Alzheimer's disease are regarded as future candidates for brain tissue transplantation. ${ }^{1}$ Proponents of brain tissue transplantation argue in general that compared to transplantation of the whole brain, transplantation of fragments of tissue

\section{Key words}

Brain tissue transplantation; personal identity; brain identity; "ethics of the brain". does not affect the identity of a person. Opponents argue that inserting tissue into the brain of a person must necessarily alter the identity of that particular $\overrightarrow{-}$ person - the brain is considered to be the carrier as $\nexists$ well as the substrate of personal identity. Hence dis-응 tinguishing between preservation and alteration of personal identity may be central to any ethical evalu- $\frac{c}{\frac{c}{\sigma}}$ ation of brain tissue transplantation.

The purpose of this article is to discuss some of the $\vec{\varphi}$ "standard" arguments with regard to personal identity $\mathscr{\&}$ which are used both by proponents and opponents. of brain tissue transplantation. These "standard" arguments are used against the backdrop of different philosophical presuppositions by proponents and opponents. The different philosophical presup- $\frac{\mathrm{Q}}{\varnothing}$ positions are examined with regard to clinical and $\cong$ neurophysiological evidence. The conclusion is that $\overrightarrow{\overrightarrow{0}}$ determining criteria for brain identity as well as 3 defining the relationship between brain identity and personal identity will be central to any evaluation of personal identity in brain tissue transplantation.

\section{Standard arguments}

FIRST ARGUMENT: NATURE OF THE TRANSPLANT Proponents argue that transplantation of the whole brain or of entire lobes, as has already been done in monkeys, ${ }^{23}$ will alter brain identity and therefore $\frac{T}{0}$ personal identity as well. ${ }^{4}$ Brain tissue transplantation, by contrast, only restores brain functions by $\mathcal{N}$ replacing degenerated cells with healthy functioning $N$ ones. The inserted cells affect neither brain identity N nor the brain-person relationship. Hence "original" $\omega$ brain function as well as personal identity are preserved. 5

For this reason, in current practice, only cells, but $\stackrel{\overparen{D}}{\oplus}$ not entire lobes or whole brains, are allowed for use $\underset{T}{+}$ for transplantation. ${ }^{5}$ Moreover degenerated cells, ${ }_{0}^{\circ}$ which are replaced by the transplant, should be $\mathbb{\Phi}$ strictly confined to a locally circumscribed area in $\frac{\rho}{\mathbb{Q}}$ the brain on the neuroanatomical as well as on the $\frac{0}{\sigma}$ neurochemical level. ${ }^{6}$

Opponents argue that the nature of the transplant $\delta$ makes no difference with regard to personal identity. Whole brains and lobes as well as cells have to beçํำ regarded as foreign material which alter identity of $\stackrel{?}{\rightleftharpoons}$ 
the brain. Due to the fact that the brain has to be considered as the substrate of the person, alterations of brain identity necessarily entail alterations of personal identity. ${ }^{7}$

From a medical perspective, such a view is supported by immunological mechanisms: the immune system distinguishes self and non-self only on the basis of the quality of the inserted material, whereas the quantity of inserted material is largely irrelevant. ${ }^{8}$ Even if the quantity of foreign inserted material is small, the immune system may reject it. Thus, from a strictly immunological perspective, there appear to be no differences between brain tissue transplantation and brain transplantation. Furthermore, the immunological situation in brain tissue transplantation still remains unclear: some authors argue in favour of therapy with immunosuppressive drugs in brain tissue transplantation. Others doubt whether the brain shows the same mechanisms of immunological rejection as other organs. ${ }^{9}$ As with immunological mechanisms, the underlying pathological processes do not distinguish between cell transplants and lobe transplants. In either case, the transplant may be affected by the underlying pathological processes within the recipient brain. ${ }^{10}$ From a philosophical perspective, opponents presuppose "strict" identity between brain and person so that even inserting a small number of new cells within the brain necessarily affects personal identity. In contrast, proponents imply a rather "loose" identity between brain and person so that not every change of "brain identity" necessarily entails an alteration of personal identity.

\section{SECOND ARGUMENT: COMPARISON WITH}

\section{PSYCHOSURGERY}

Proponents consider brain tissue transplantation only as a replacement of degenerated cells in order to restore an already impaired function. Thus personal identity is repaired and preserved rather than altered. ${ }^{11}$ Psychosurgery removes whole lobes and complete functions. ${ }^{3}$ These functions may be closely linked with personal identity and if the former are lost the latter must necessarily be altered. Hence brain tissue transplantation cannot be compared with psychosurgery because inserting cells is something fundamentally different from removal of whole lobes. ${ }^{5}$

The problem of the distinction between insertion and removal is central to this argument: insertion of foreign material into the brain is often considered to cause an alteration of personal identity, while removal of parts of the "original" brain, for example tumour removal, is not. Even though the quantity of the affected material may be much smaller in brain tissue transplantation than in tumour removal, only the former is often linked with alterations of personal identity. Proponents agree on the principal difference between brain tissue transplantation on the one hand and removal on the other hand. But they "invert" the argument by stating that restoration of lesioned functions is something essentially different from removal of pre-existing functions, only the latter causing an alteration of personal identity.

Opponents argue that there is such a close relationship between inserting foreign material and personal identity that brain tissue transplantation has to be regarded as an "insertion of a new personal identity". ${ }^{7}$ Psychosurgery may alter personal identity but it does not create or insert a new personal identity. Hence opponents emphasise that insertion of foreign material is more likely to result in alteration of personal identity than removal of own material.

Proponents and opponents rely on different definitions of "personal identity": proponents point out that substitution of lesioned functions does not alter personal identity qualitatively. Opponents on the other hand imply a numerical sense of personal identity by linking any foreign material necessarily to new personal identity. Consequently opponents such as Linke assume that inserting fetal material into the brain is likely to result in an alteration and enhancement of personal identity. ${ }^{7}$

From a clinical perspective no studies about possible alterations of personal identity in brain tissue transplantation are known to us. As is the case with drug therapy, it may be expected that, due to improvement of motor functions, the patients themselves experience a restoration of personal identity rather than an alteration. Psychosurgical patients, in contrast, showed alterations, some severe, of their personal identity. ${ }^{3}$

THIRD ARGUMENT: COMPARISON WITH TRANSPLANTATION OF OTHER ORGANS

Proponents consider the brain the central organ for constituting personal identity. Consequently transplantation of the whole brain should not be allowed. ${ }^{12}$ Conversely brain tissue transplantation should be compared with transplantation of other organs rather than with transplantation of the whole brain. Inserting tissue into the brain as well as inserting organs into the body should be regarded as replacements of lesioned functions. Whereas transplantation of the whole brain cannot be regarded only as a replacement of lesioned functions but, in addition, as an insertion of a new personal identity.

From a neurophysiological perspective, such a view is supported by the functional organisation of the brain: not every region or function within the brain (for example, brain stem, etc) seems to be closely related to personal identity. There are brain areas and functions (brain stem, cerebellum, hormonal, immunological, etc) where insertion of foreign material would probably not interfere with personal identity. Whereas other regions within the brain, for example, the frontal lobe and the limbic system, etc are much more closely connected with the personality and the identity of a particular 
person. Hence, evaluation of personal identity in brain tissue transplantation depends strongly on the "functional localisation" of the insertion of tissue within the brain of the recipient.

Opponents rely on the "strict" identity between brain identity and personal identity: if one argues that the main difference between the brain and other organs consists in the fact that the brain is the central organ for personal identity every alteration of brain identity must necessarily imply an alteration of personal identity. Both insertion of foreign tissue and insertion of a new brain must thus necessarily alter brain identity and consecutively personal identity as well. Hence, relying on the special role of the brain compared to other organs one can no longer assume a qualitative difference between brain tissue transplantation and brain transplantation with regard to personal identity.

From a philosophical perspective proponents and opponents rely on different meanings of the term "brain identity": proponents imply a "qualitative" identity of the brain, which is only affected in brain transplantation. Insertion of tissue does not alter but restores functions of the brain and therefore its qualitative identity. Opponents, in contrast, rather rely on a "numerical" sense of brain identity inferring a new brain identity from the insertion of foreign brain material. Thus, by contrast with qualitative brain identity, with its emphasis on brain functions, the definition of numerical brain identity relies on the importance of the material and the brain structure. However, both proponents and opponents, may have a point in arguing, on the one hand, for preserving a qualitatively understood identity of the brain and, on the other, for an alteration of a numerically conceived identity of the brain.

\section{FOURTH ARGUMENT: RESTORATION OF MOTOR} FUNCTION

Proponents argue that brain tissue transplantation in Parkinson's disease should merely be regarded as a restoration of motor function. Implanted cells are only inserted in strictly circumscribed motor areas, ie the caudatum and/or the putamen, ${ }^{6}$ which do not affect psychological functions or personal identity at all. ${ }^{13}$ Due to surgical manipulations within the respective brain areas some kinds of psychosurgery interfere with psychological functions and consequently with personal identity as well. ${ }^{14}$

Only Parkinsonic patients who have not suffered from any psychological alterations are considered for brain tissue transplantation. ${ }^{11}{ }^{15}$ So far there have not been any reports of major psychological alterations following transplantation. ${ }^{16}$

Opponents argue that such a strict distinction between motor and psychological functions is not possible: given a person's facial expression or melody of speech, separating motor and psychological, ie cognitive and affective functions seems to be impossible. ${ }^{7}$ Furthermore, areas in the brain (cerebellum,

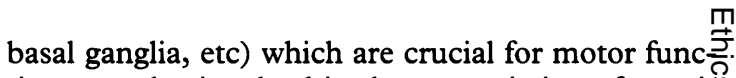
tions are also involved in the transmission of cogni ? tive and emotional functions. The functionat organisation of the brain itself therefore does nof allow a strict separation between psychological and motor functions. Hence insertion of tissue in Parkinson's disease does not solely replace lesione $\bar{b}$ motor functions but may reorganise psychologicaP functions as well.

From a clinical perspective there may be evidences for both views: on the one hand there are certainkinds of movement (extension and flexion of legs) which are rather mechanistic and simple so that they are more or less unrelated to psychological functD tions. On the other hand there are more complexin movements (gestures, facial expression, etc) whichio are strongly modulated by and closely related to psychological functions.

FIFTH ARGUMENT: RELATIONS BETWEEN BRAIN AND BODY

Proponents argue that transplantation of the wholed brain implies either a switch of the body or of the brain: new relations between the "new" brain and the "original" body or vice versa have to bi established. ${ }^{14}$ If brain and body are related in a new way, personal identity may be altered as well. ${ }^{7}$ Brain tissue transplantation does not alter relation\$ิ between brain and body because both remain essentially the same. ${ }^{5}$ Therefore insertion of tissue may preserve personal identity.

From a medical perspective brain and body are closely interrelated through hormonal, immuno logical, nervous, etc functions: hormones, antibodies and neuronal impulses can be produced in the body and may affect brain functions or vice versa $\sigma$ Whether relations between brain and body are altered in brain tissue transplantation must remaim. open: so far hormonal alterations have not occurred in Parkinsonic patients treated with tissue transplano tation whereas the immunological situation is still unclear (see above).

Opponents of brain tissue transplantation argue that insertion of foreign tissue necessarily alters identity of the brain. Consequently the "new" brain must have different relations with the body than the "old" brain. Hence every manipulation within thebrain necessarily implies an alteration of the relations between brain and body and therefore to personal identity as well. ${ }^{7}$

From a philosophical perspective proponents and opponents rely on different definitions of the relations between brain and body with regard to personal identity: proponents assume that persona原 identity is not necessarily predicated upon brain $\frac{2}{\sigma}$ body relations. Even if the latter undergo changes the former can remain the same. Therefore preserva tion of brain-body relations may only be a necessarys not a sufficient, condition for personal identity을 Opponents assume that personal identity is closely 
linked to brain-body relations so that alterations of brain-body relations necessarily imply alteration of personal identity. Thus, by contrast with the view held by proponents, preservation of brain-body relations has to be regarded as a sufficient condition for personal identity.

\section{SIXTH ARGUMENT: RELATIONS BETWEEN BRAIN AND} MIND

Proponents argue that transplantation of the whole brain implies not only a replacement of the brain but an exchange of minds as well. ${ }^{4}$ Brain tissue transplantation preserves the "original" brain and the "original" mind as well. Hence brain tissue transplantation neither alters brain-mind relations nor personal identity. ${ }^{5}$

From a philosophical perspective there are different positions on the relations between brain and mind: some philosophers ${ }^{17}$ explain mental states by referring solely to the brain. Other philosophers ${ }^{18}$ explain mental states instead by means of psychological functions. In addition there are positions ${ }^{19}$ which reject any physicalistic or psychological explanations of mental states. Depending on the philosophical presuppositions about the relations between brain and mind one might evaluate the influence of brain tissue transplantation on these.

From a clinical perspective there are so far no reports about alterations of phenomenal experiences, ie mental states, in Parkinsonic patients after transplantation. ${ }^{13}$

Opponents argue that every manipulation within the brain must necessarily affect the mind ${ }^{7}$ : motor functions may be fully restored but phenomenal experiences, ie mental states, may be different. Foreign material within the brain must necessarily alter the mind. Consequently brain-mind relations as well as phenomenal experiences of that person are altered. Even an "inversion of phenomenal experiences" such that the person may see the colour red while experiencing blue cannot be fully excluded. ${ }^{20}$

\section{SEVENTH ARGUMENT: PSYCHOLOGICAL FUNCTIONS}

Proponents argue that transplantation of the whole brain implies replacement not only of the brain, but of psychological functions as well. After transplantation memories and thoughts are no longer those of the "original" person but those of the donor so that psychological continuity is disrupted. Therefore the "new" person is no longer identical with the "old" person. Brain tissue transplantation, in contrast, as a substitution of lesioned and degenerated cells instead restores continuity of psychological functions. ${ }^{5}$ Hence, brain transplantation and brain tissue transplantation cannot be considered comparable with regard to psychological continuity.

Examination of Parkinsonic patients after transplantation has shown no major psychological abnormalities so far. Thus there may be no disruption of psychological continuity in "motor transplants". In the case of "psychological transplants" the question of disruption or preservation of psychological continuity must remain open: the difference between replacement and alteration may be less clear-cut here than in the case of "motor transplants" because restoration of psychological continuity may entail disruption of psychological continuity. Moreover there have so far been no reports of patients because such transplantations have not been considered yet.

Opponents argue that brain tissue transplantation, while restoring psychological functions, may disrupt psychological continuity ${ }^{7}$ : memory functions may be fully restored but the contents of the memory may be different from before. Will I have the memories of my neighbour when his memory tissue is transplanted into my brain? Will the resulting person have the identities of both persons, its "original" identity or the identity of the neighbour? Hence, transplantation of psychological functions may lead to dissociation between psychological contents and personal identity, which normally are closely intertwined.

From a neurophysiological perspective it is rather improbable that isolated psychological functions may be transplanted in small pieces of tissue from one person to another: though memory is strongly associated with the function of the hippocampus, this anatomical structure is itself functionally regulated by other cortical areas so that insertion of a hippocampus would not be sufficient for full restoration of memory functions. Moreover, evaluation depends on the presupposed model of brain organisation with regard to psychological functions: authors like Fodor ${ }^{21}$ rely on a cognitive model where psychological functions are localised within separate "modules" such that the brain functions like a computer with different modules. Relying on such a model, transplantation of "modules", for instance of particular psychological functions, seems not impossible. Other authors, such as PM Churchland ${ }^{22}$ rely on a connectionist model which regards the brain as a functional neuronal network where changes in one area alter functions of the whole neuronal network. Relying on this "neuronal network model", transplantation of isolated psychological functions and contents seems rather unlikely because they are considered to be dependent on the functioning of the whole neuronal network.

From a philosophical perspective the relation between psychological functions and personal identity is central to this argument: $D$ Parfit ${ }^{18}$ regards psychological continuity as a necessary as well as a sufficient condition for personal identity. Others, like R Swinburne, ${ }^{19}$ do not consider psychological continuity as a necessary and sufficient criterion for personal identity but rather identify persons with their mental states which are neither reducible to psychological functions nor to physical brain states. Hence, among philosophers there is no 
general agreement about the necessary and sufficient criteria for personal identity.

\section{EIGHTH ARGUMENT: NEUROPHYSIOLOGICAL} PROPERTIES

Proponents argue that transplantation of the whole brain necessarily implies transplantation of neurophysiological properties. After transplantation, the recipient shows these neurophysiological properties and consequently the personal identity of the donor. Brain tissue transplantation, by contrast, only restores neurophysiological properties in such a manner that neither "neurophysiological brain identity" nor personal identity are affected. ${ }^{11}$

So far it remains unclear how the inserted tissue functions ${ }^{115}$ : some authors assume that the inserted tissue functions like a "biological mini-pump" serving only to excrete the lacking transmitter dopamine. This is supported by the fact that other transmitter systems which are altered in Parkinson's disease as well (serotonin, glutamate, noradrenaline, acetylcholine) are not affected by the transplant itself. ${ }^{15}$ Others claim that full restoration of neuronal connectivity is necessary for complete recovery from Parkinsonic symptoms. ${ }^{1}$ After transplantation, Parkinsonic patients show improvement of their motor symptoms but no complete recovery: they need less medication, respond better to them and show improvement of dopaminergic function in positron emission tomography (PET) studies. $^{1623}$ Though there may be substitution on the neurochemical level, ie of the transmitter dopamine, functional connectivity may not be fully restored. Otherwise a complete recovery of Parkinsonic symptoms would be expected.

Opponents argue that even if neurophysiological properties are fully restored the overall functioning of the brain may be different from before. The inserted tissue may be neuronally and functionally integrated into the neuronal network in a different way so that the functional organisation of the brain is altered. Hence restoration of neurophysiological properties might not necessarily lead to preservation of functional brain organisation.

From a philosophical perspective, relations between neurophysiological brain properties and personal identity are central to this argument: a philosopher like Thomas Nagel ${ }^{17}$ claims that the brain must be considered a necessary and sufficient condition for personal identity because "I am my brain". According to such a view every insertion of tissue into the brain must necessarily be accompanied by alterations of personal identity. Other philosophers, for example B Williams, ${ }^{24}$ argue that identity of the brain can only be considered a necessary condition for personal identity. Due to the fact that parts of the brain can be removed without any alterations of personal identity, the brain cannot be regarded as a sufficient condition. According to this view, brain tissue transplantation may not necessarily interfere with personal identity because the latter is onl $\overrightarrow{x_{0}}$. partially dependent on the brain. Another philos? sopher, D Parfit, ${ }^{18}$ even asserts that personal identit: is logically independent of the brain: the brain is cons? sidered to be morally the cause of psychological cone tinuity, ie of personal identity, which is the effect This effect could, in principle, be generated by othe causes, for example, a computer, so the brain cannoe be a necessary condition for personal identityen According to Parfit's view the material of the cause, if original brain material, inserted tissues, etc, does not matter for personal identity as long as the effects, i $\vec{w}$ psychological continuity, are the same.

\section{Brain identity and personal identity}

The "standard" arguments showed that determination of brain identity, distinction between preserva $A$ tion and alteration of brain identity and the relation between brain, psyche, and mental states in personat identity have to be considered as central problems in brain tissue transplantation.

\section{IDENTITY OF THE BRAIN: STRUCTURAL AND}

FUNCTIONAL IDENTITY

Different levels of brain identity should be distine guished: the brain shows a particular anatomicat structure, ie structural identity, as well as neuro음 physiological and psychological functions, ie func요 tional identity. ${ }^{25}$ Structural brain identity does no₹ necessarily imply functional identity, either neuroō physiologically or psychologically, and vice versa.

Patients may show severe anatomo-structura稏 lesions without any functional alterations. On the. other hand there are "functional diseases", fo example certain psychiatric disorders, ${ }^{25}$ which cannof be put down to structural lesions. In the case of brain tissue transplantation, relations between structura and functional brain identity remain unclear: as demonstrated in brain-imaging studies (PET), struc tural lesions, ie nigrostriatal dopaminergic tracts, are restored after transplantation. ${ }^{1623}$ Nonetheles 8 Parkinsonian patients do not show full remission of their symptoms so that functional brain identito cannot be fully restored. ${ }^{1023}$ Therefore restoration off structural brain identity in brain tissue transplantaru tion does not necessarily imply preservation of func tional brain identity.

Structural and functional brain identity may faff together but they can dissociate from each other well. ${ }^{12}$ As has been shown in brain tissue transplantation, structural brain identity cannot be regarde $\$$ as a sufficient condition for functional brain identity $\vec{p}$ Consequently "anatomo-structural localisation $\frac{\Phi}{\Phi}$ within the brain does not necessarily coincide with "functional localisation": the smallest deviations (some of a micrometre) of the localisation of insertion of tissue may lead to major function differences. ${ }^{13}$ Furthermore, the best localisation insertion of tissue in Parkinson's disease is sti 
unclear. Some neurosurgeons insert tissue into the nucleus caudatus whereas others prefer the putamen. ${ }^{15} 23$

Moreover, with regard to functional brain identity individual and environmental influences have to be considered. Functional brain identity develops in close relation with the environment of the respective individual person. A ballet dancer, for example, may show much more individuality and ability with respect to fine-grained movements than a philosopher who is trained in abstract thinking. Such different abilities are probably reflected in the intensity and the expansion of activated areas within the brain. Whether insertion of tissue would fully restore individuality of movements in a ballet dancer must remain open. Individuality of brain function can also be observed in psychiatric patients: schizophrenic patients with the same symptoms often react in a totally different way to the same neuroleptic medication.

\section{DISTINCTION BETWEEN PRESERVATION AND}

\section{ALTERATION OF BRAIN IDENTITY}

Removal of a tumour, neuroleptic medication, psychosurgery and brain tissue transplantation do alter the brain either structurally and/or functionally. Where is the border between preservation and alteration of brain identity? Is there a "threshold of disruption"? ${ }^{26}$

If some parts of a bicycle are replaced by new ones, one identity of the bicycle is preserved. ${ }^{26}$ Even if the new parts differ from the former ones, the identity of the bicycle is not necessarily disrupted. Similarly, in the case of brain tissue transplantation, replacement of degenerated motor tissue does not necessarily disrupt brain identity. Even if different material is successfully inserted, for example microelectrodes in Parkinson's disease, ${ }^{27}$ functional brain identity may be restored. Hence, rather than the quantity and quality of inserted material, it is the "functional localisation" of the insertion which seems to be most important when attempting to distinguish between preservation and alteration of brain identity.

Moreover, the distinction between preservation and alteration of brain identity has to consider individuality of brain function (see above) as well: the same insertion of tissue may preserve functional brain identity in one person whereas it may alter functional brain identity in another. Higher cortical functions, ie psychological functions, in particular, show a high degree of individuality concerning their functional localisation within the brain. Hence, future applications of brain tissue transplantation in psychological diseases will have to take such individuality of brain functions into account.

Future developments of possible transplants are central for full restoration of functional brain identity: currently fetal tissue is used for brain tissue transplantation in Parkinson's disease. Because of ethical problems concerning the use of fetal tissue $e^{28}$ other sources of transplants are being developed. These include genetically modified cells which excrete the missing transmitter, ie dopamine, and/or certain nerve growth factors. ${ }^{29}$ Moreover, the combination of brain tissue transplantation with certain forms of gene therapy are being considered. ${ }^{10} 1529$ Concerning future transplantation of isolated cells and genes, the question of the importance of cell-identity and DNAidentity ${ }^{8}$ for functional brain identity may be raised: restoration of dopaminergic cells within the brain in Parkinson's disease does not necessarily lead to full remission of symptoms (see above), so cell-identity can be regarded neither as a necessary nor as a sufficient condition for functional brain identity. In order to avoid immunological rejection, DNA-identity has to be considered as a necessary condition for functional brain identity. Taking individual functional variability of the brain into account (see above), DNA-identity cannot be considered a sufficient condition for functional brain identity.

\section{PERSONAL IDENTITY: BRAIN, PSYCHE AND MENTAL} STATES

People have mental states as well as psychological functions, and they have a brain and a body with physical properties. Thus, criteria for necessary and sufficient conditions for personal identity with regard to the mind-body/brain relation can either be physical, psychological or mental. ${ }^{30}$

Some philosophers ${ }^{19}$ assume that personal identity is closely related to mental states which by themselves can neither be reduced to psychological functions nor to neurophysiological properties. In this context the question of the possibility of transplantation of mental states would arise: does insertion of "motor tissue" imply insertion of mental states? According to this position transplantation of tissue should not include mental states because these are non-reducible. Transplantation of tissue with solely neurophysiological properties should therefore not affect personal identity. Hence, according to this view, investigation of mental states is central to evaluating personal identity in brain tissue transplantation.

Another position, defended by $\mathrm{D}$ Parfit, ${ }^{18}$ considers psychological continuity as a sufficient condition for personal identity: according to this view, personal identity is preserved if patients show the same psychological contents before and after brain tissue transplantation. Clinically, major psychological changes in patients after transplantation have not been observed so far. ${ }^{13}$ It is not clear whether future transplantation of psychological functions such as memory, cognition, etc, might alter psychological contents and therefore psychological continuity as well. Psychosurgery showed that removal of brain areas which are closely related to psychological functions (frontal lobe, limbic system, etc) could lead to disruption of psychological continuity. ${ }^{3}$ 
Whether insertion of brain tissue can be compared in its impact on psychological continuity and personal identity to psychosurgery remains questionable (see above).

Other philosophers, for example $T$ Nagel, ${ }^{17}$ emphasise the central role of the brain as a necessary and sufficient condition for personal identity ("I am my brain"). If personal identity is so closely associated with brain identity every insertion of tissue must be regarded as an alteration of personal identity. Hence, according to this view, the question for personal identity in brain tissue transplantation can fully be answered by brain identity.

\section{Conclusion}

I have discussed "standard" arguments used by proponents in favour of preservation of personal identity as well as by opponents in favour of alteration of personal identity in brain tissue transplantation. I have demonstrated that proponents and opponents use the same arguments but with different underlying presuppositions. These presuppositions concern the meaning of the term "identity", either numerical or qualitative, the definition of brain identity, either structurally or functionally, as well as the relations between mental states, psychological functions and neurophysiological properties. Neurophysiological, clinical and philosophical evidence for the different underlying presuppositions have been discussed. It can be concluded that evaluation of personal identity in brain tissue transplantation should not only rely on "standard" arguments but on neurophysiological, clinical and philosophical evidence as well.

Georg Northoff, $M D, P h D$, is Senior Resident in the Psychiatry Department of the University of Frankfurt, Germany.

\section{See editorial page 131.}

\section{References}

1 Björklund A. Neural transplantation - an experimental tool with clinical possibilities. Trends in Neuroscience 1991; 14: 319-3222.

2 White RJ, Wolin LR, Massopust L, Taslitz N, Verdura J. Cephalic exchange transplantation in the monkey. Surgery 1971; 70: 135-9.

3 Valenstein ES. Great and desperate cures: the rise and decline of psychosurgery and other radical treatments of mental illness. New York: New York University Press, 1986.

4 O'Shea B. Brain Transplants: myth or monster? British Fournal of Psychiatry 1990; 157: 302.

5 Hoffer BJ, Olson L. Ethical Issues in brain-cell transplantation. Trends in Neuroscience 1991; 14: 384-8.

6 Nikkah G, Sauer H. Transplantation von Gehirnzellen Vision oder Realität? Deutsches Arrteblatt 1992; 3: 76-81.

7 Linke DB. Hirnverpflanzung. Die neue Unsterblichkeit auf Erden. Hamburg: Rowohlt, 1993.
8 Munzer S. Transplantation, chemical inheritance and the identity of organs. British fournal of Philosophy and? Science 1994; 45: 555-70.

9 Freed WJ, Poltorak M. Comments on brain tissue transplantation without immunosuppression. Archive? of Neurology 1991; 48: 259-61.

10 Lindvall $O$. Prospects of transplantation in humans

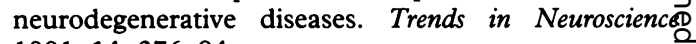
1991 ; 14: 376-84.

11 Kupsch A, Sauer H, Oertel WH. Transplantation von Dopamin-herstellenden Nervenzellen: Eine neueb Therapiestrategie gegen das idiopathische Parkinson Syndrom. Nervenarzt 1991; 62: 80-91.

12 Green MB, Wikler D. Brain death and personaR identity. Philosophy and Public Affairs 1980; 9: 105-33.

13 Widner $\mathrm{H}$. Brain tissue transplantation. Lecture at the congress, Neuroworlds. Düsseldorf, 1993 Nov: 3-4.

14 Denman C. Ethics of "brain transplants". Britis Fournal of Psychiatry 1990; 157: 625.

15 Goetz CG, Long De $M$, Penn RD, Bakay $\mathrm{RA}_{\mathrm{O}}^{+}$ Neurosurgical horizons in Parkinson's disease? Neurology 1993; 43: 1-7.

16 Turner DA, Kearny W. Scientific and ethical concern§ in neural fetal tissue transplantation. Neurosurger ${ }^{\circ} \mathrm{D}$ 1993; 33: 1031-7.

17 Nagel T. The view from nowhere. Oxford: Oxfordo University Press, 1986.

18 Parfit D. Reasons and persons. Oxford: Oxford University Press, 1984.

19 Swinburne R. The evolution of the soul. Oxford: Oxfors University Press, 1986.

20 Northoff G. Qualia im Knotenpunkt zwischen Leik๊ und Seele: Argumentative Dilemma in der gegenwärti gen Diskussion über die Subjektivität mentale $\overrightarrow{\vec{D}}$ Zustände. Fournal of General Science 1995; 26? 269-295.

21 Fodor JA. The modularity of mind. An essay on facult psychology. Cambridge, Mass: MIT Press, 1983.

22 Churchland PM. Matter and consciousness. A contempo rary introduction to the philosophy of mind. Cambridge Mass: MIT Press, 1988.

23 Sawle GV, Bloomfield PM, Björklund A尺े Transplantation of fetal dopamine neurone in Parkinson's disease: PET 18F 6 L-Fluor-Dopa Studies in two patients with putaminal implants. Annals of Neurology 1992; 31: 166-73.

24 Williams B. Problems of the self. Cambridge: Cambridge University Press, 1973.

25 Caine CD, Joynt RJ. Neuropsychiatry again. Archives of Neurology. 1986; 43: 325-7.

26 Elliot R. Identity and the ethics of gene therapy Bioethics 1992; 7: 27-40.

27 Jean S. Chronic electrical stimulation of the pose teroventral pallidum for a better control of manf symptoms of Parkinson's disease. Eleventh Internationa Symposium on Parkinson's Disease. Rome, Italy, $1994^{+}$ Mar 26-30.

28 Boer $G$ on behalf of Nectar. Ethical guidelines for the्षे use of human embryonic or fetal tissue. Fournal of Neurology 1994; 242: 1-13.

29 Northoff G. Hirngewebstransplantationen - eine aktuelle Übersicht. Ethik in der Medizin 1995; $7 \dot{8}$ 87-92.

30 Rorty A. Indentities of persons. Los Angeles: Californt University Press, 1976. 